\title{
THE PAINFUL ARC SYNDROME \\ CLINICAL CLASSIFICATION AS A GUIDE TO MANAGEMENT
}

\author{
Lipmann Kessel and Michael Watson, London, England
}

\section{From the Institute of Orthopaedics and Royal National Orthopaedic Hospital, London and Stanmore}

\begin{abstract}
Ninety-seven patients suffering from painful arc syndrome of the shoulder were studied. Local anaesthetic and radiographic contrast investigations were carried out. One-third of the patients had lesions in the posterior part of the rotator cuff which resolved after injections of local anaesthetic and steroid. One-third had anterior lesions in the subscapularis tendon: almost all resolved under the same regime but two required division of the coraco-acromial ligament. The remaining third had lesions of the supraspinatus tendon, usually associated with degeneration of the acromio-clavicular joint: most of these failed to gain relief from the local anaesthetic and steroid. Twenty-two operations were performed either by a transacromial or by a deltoid splitting approach. Excision of the outer end of the clavicle and division of the coraco-acromial ligament abolished the pain in most cases.
\end{abstract}

"Painful shoulders form an important part of orthopaedic practice, but their obscurity, uncertain prognosis, and the fact that they present so few definite signs and symptoms, render their classification into types difficult on clinical grounds." (Burns and Ellis 1937.)

Compared with the enormous strides made in orthopaedic surgery in the thirty years since that was written, there have been few important advances in our understanding of common soft-tissue disorders of the shoulder. This paper is an attempt to fill the gap in the hope that further work will lead to a clearer understanding of a common clinical problem.

\section{PATHOLOGY}

The painful arc syndrome is characterised by pain referred to the lateral aspect of the upper arm in the region of the bulk of the deltoid muscle and its insertion. The pain may be felt at rest, characteristically more so at night, and is typically exacerbated within a certain arc of movement. A painful arc between 60 and 120 degrees of abduction indicates some disorder of the subacromial region. An arc of pain which becomes increasingly severe to full elevation of the arm at 180 degrees indicates a disorder of the acromio-clavicular joint (Fig. 1).

The subacromial region comprises a functional joint between the acromio-clavicular and coraco-acromial arch above and the tendinous cuff and tuberosities of the humerus below. The intervening large subacromial bursa acts as the joint cavity. Affections in this region give rise to the most common and the most characteristic disorders of the shoulder girdle. The syndrome can be

caused by several different lesions, all of which have one thing in common: there is loss of the normal clearance between the arch above and tuberosities below during

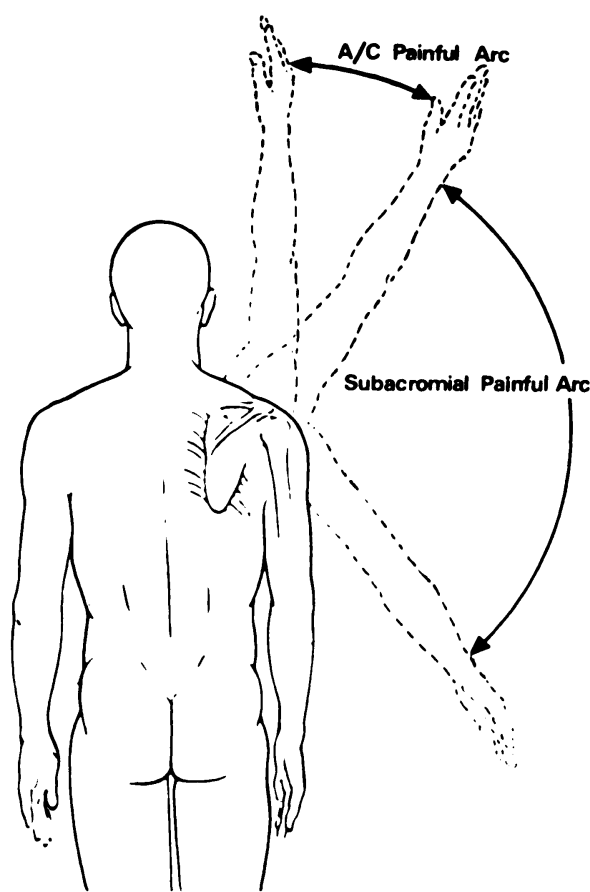

FiG. 1

Sketch to indicate the painful arcs of abduction arising from the subacromial and acromioclavicular joints.

abduction of the arm, or loss of the normal gliding of the bursal walls.

This article is an attempt to analyse the patterns of the painful arc syndrome in order to provide a guide to 
management; it does not attempt to explain or detail the variety of pathological changes which occur in this region. With regard to pathology, although most authors (Codman 1934; Bosworth 1940; McLaughlin 1945; Arm-

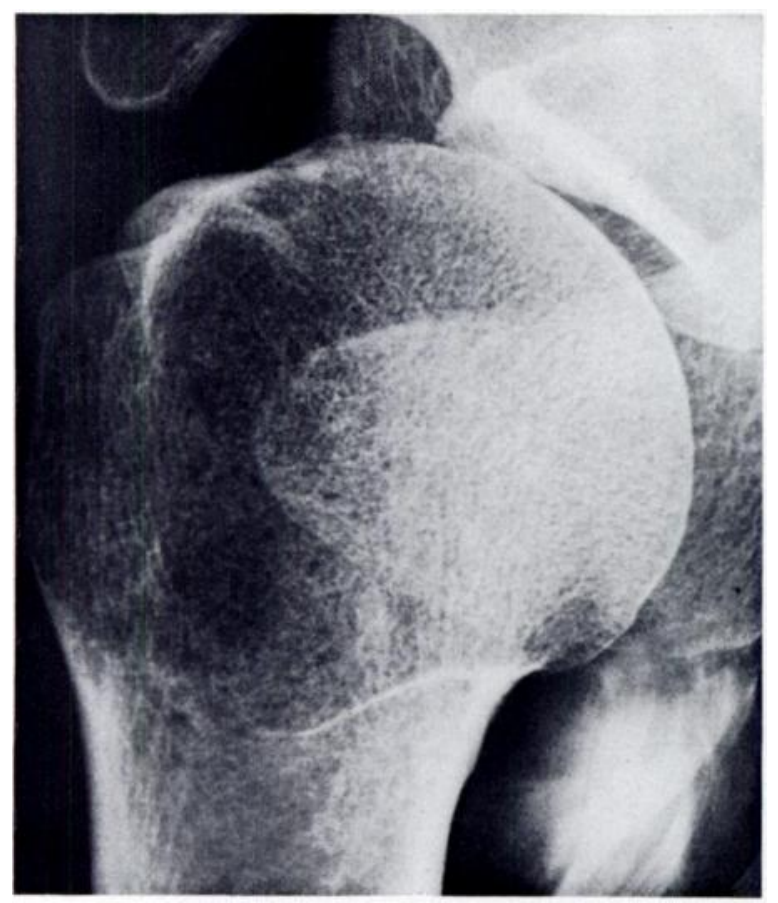

Fig. 2

Hypaque has been injected into the source of pain in a posterior type lesion. In the axial view, the medium can be seen lying in the posterior part of the rotator cuff just lateral to the angle of the acromion.

strong 1949) are agreed that the supraspinatus tendon harbours almost all the lesions, we have found this true in only about one-third of our patients. Degenerative change of the acromio-clavicular joint in this context has not been taken into account until recently (Neer 1972), but a glance at our Tables will show that we believe it to be important.

Whatever the details of the pathology may be, the final common denominator is a set of symptoms and signs, usefully called the painful arc syndrome (Adams 1955; Kessel 1967).

We have tried to analyse why it is that a proportion of patients become pain-free within a few weeks after only simple local therapy, whereas others suffer intractable pain and require operative intervention after a variable length of time. Is it possible to identify those patients early in the course of their complaint who are destined to have intractable pain? And if so, is it possible to offer them an early operation with a reasonable chance of success?

\section{CLINICAL MATERIAL}

Over a two-year period all patients with a painful arc syndrome attending a shoulder clinic have been studied. The presumed site of the lesion causing the pain was identified as accurately as possible by palpation, and by the use of local anaesthesia and radio-opaque contrast medium. The progress of each patient was then followed until a steady state was reached. An attempt was made to correlate the natural history of the complaint with the site of the lesion, and wherever possible its pathology. The response to various therapeutic manoeuvres was also examined in this light.

Ninety-seven patients were studied and treated; their average age was fifty-one years and the male: female ratio was 1 to $1 \cdot 8$. Patients with typical acute calcific tendonitis who had severe sudden pain with virtually no shoulder movements were excluded from the study, as were patients with acute rupture of the tendon of the long head of biceps or rotator cuff. None of these present with a typical painful arc syndrome. The commonest original mistaken diagnosis was pain due to cervical spondylosis:

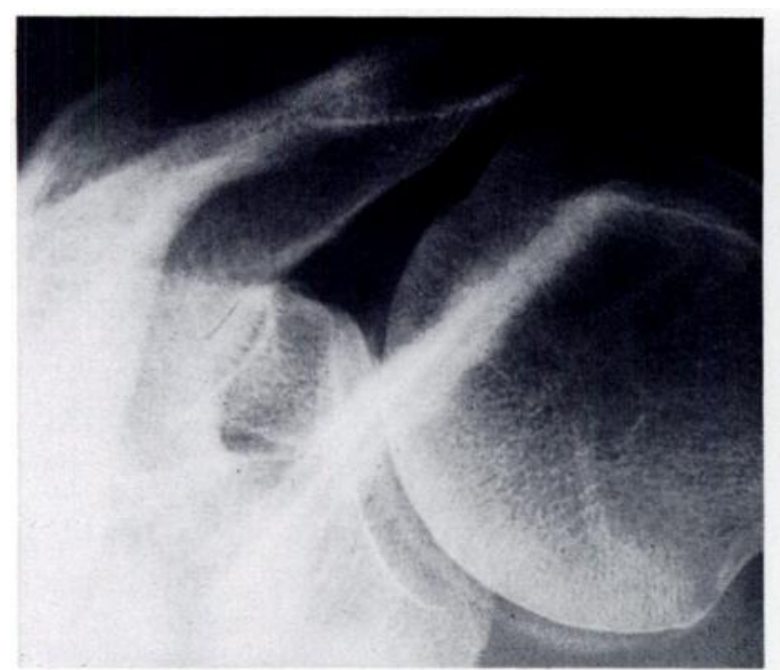

Fig. 3

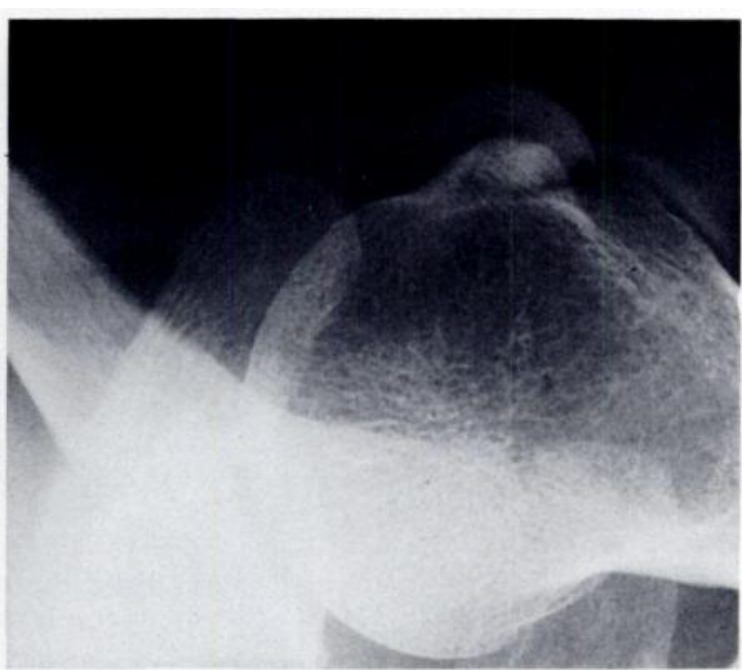

Fig. 4

This is an anterior type lesion. The medium lies in the line of the subscapularis fibres on the antero-posterior view and can be seen pooled around the lesser tuberosity on the axial view. 
this is easily distinguishable by an accurate history, because pain arising from the shoulder joints never radiates proximally to the side of the neck.

\section{IDENTIFICATION OF THE LESION}

An attempt was made to identify the site of origin of pain in each patient by injecting 1 millilitre of 1 per cent Lignocaine into the most tender area. Marked diminution in the severity of the symptoms together with the abolition of local tenderness and, in particular, loss of the painful arc during abduction, was accepted as evidence that this region harboured the painful lesion.

In a representative sample of patients the source of the pain was visualised with a radio-opaque medium, by injecting 1 millilitre of 45 per cent Hypaque through the needle that had delivered the local anaesthetic. Anteroposterior and axial radiographs were then taken with the shoulder in the degree of abduction and rotation which had been most painful. In this way it was usually possible to demonstrate both the anatomical structure from which the pain was arising and the position occupied by that structure in the posture of maximal pain (Figs. 2 to 5 ).

Tilt radiographs of the acromio-clavicular joints (Figs. 6 and 7) and tangential views of the bicipital sulcus (Fig. 8) were taken when these structures were suspected of being abnormal.

\section{TREATMENT}

All patients received steroids with a long-acting local anaesthetic, 40 milligrams of methyl prednisolone (DepoMedrone) with 5 millilitres 1 per cent Marcaine injected into the anatomical site of the source of pain. Surgical exploration of the lesion was considered only if symptoms persisted or rapidly returned after two or three injections. The operations were of two types depending on the preoperative diagnosis; either 1) excision of the outer 1 centimetre of the clavicle followed by a deltoid splitting exposure of the coraco-acromial ligament, its division and exploration of the underlying structures, or 2) an exploration of the subacromial bursa and underlying musculo-tendinous cuff.

Because deltoid-detaching incisions and sagittal acromionectomy or acromionotomy entail unacceptably long post-operative immobilisation and rehabilitation, a muscle-conserving approach was developed for these cases.

\section{THE TRANSACROMIAL APPROACH}

The development of the transacromial approach arose from a number of sources. As long ago as 1932 Fowler mentioned, but did not describe in detail, a coronal acromion-splitting approach to the torn rotator cuff; Diamond (1964) showed how the trapezius and deltoid form a continuous sheet over the acromion; McFarland

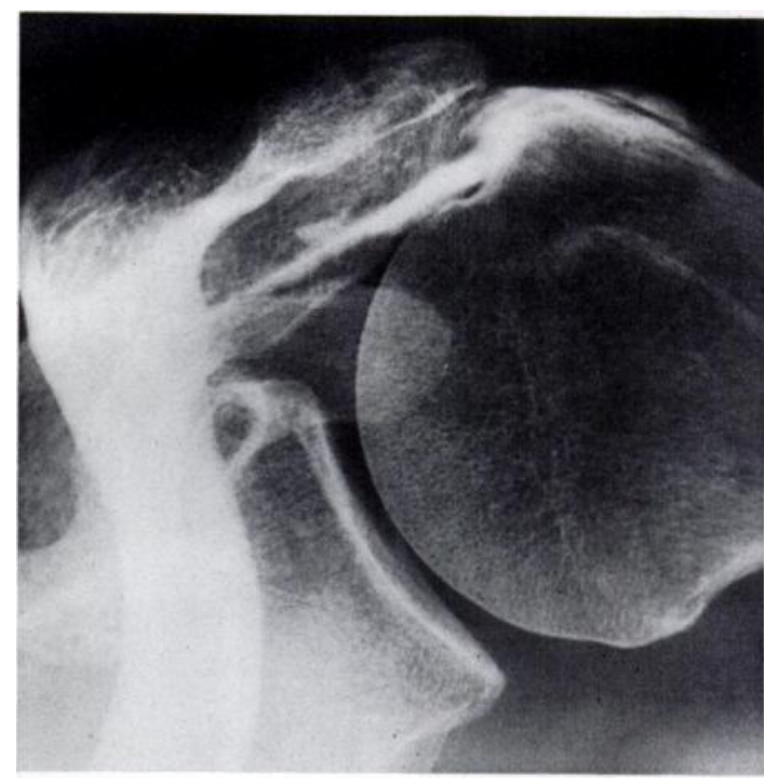

Fig. 5

An antero-posterior view of a superior type lesion. The medium lies in the supraspinatus tendon. In 90 degrees of abduction a kink is seen in the tendon where the coracoacromial ligament impinges upon it. The acromio-clavicular joint is degenerate.

and Osborne (1954) described how the hip joint could be approached without impairing the integrity of the important lateral musculature by detaching the insertion of gluteus medius in continuity with the origin of vastus lateralis in a continuous sheet with underlying periosteum. If one combines this important concept with that of Debeyre, Patte and Elmelik (1965) who divide the acromion and detach trapezius from the spine of the scapula in advancing the supraspinatus muscle laterally, one may arrive at the present operation. Trickey (1974) uses the same approach for the surgical treatment of fractures of the greater tuberosity.

Operation-The skin incision runs in the coronal plane across the middle of the acromion just behind the posterior part of the acromio-clavicular joint. The line of the incision is conveniently felt by pressing the index finger into the supraspinous fossa: the radial aspect of the finger palpates the back of the clavicle and the ulnar aspect is in contact with the spine of the scapula (Fig. 9). The incision extends 5 centimetres both proximally and distally from the acromion process (Fig. 10). Trapezius and deltoid muscles are split in the line of their fibres up to the acromion. The line of the incision across the acromion is coagulated and short flaps of conjoint aponeurosis, periosteum and bone are raised with an osteotome in continuity with the trapezius and deltoid muscles (Figs. 11 and 12). The acromion is split with an oscillating saw. Retraction of the two halves of the acromion with a self-retaining retractor exposes the subdeltoid bursa and underlying rotator cuff through a gap of some 3 centimetres (Fig. 13). By medial and lateral rotation of the arm the whole of the rotator cuff may be 


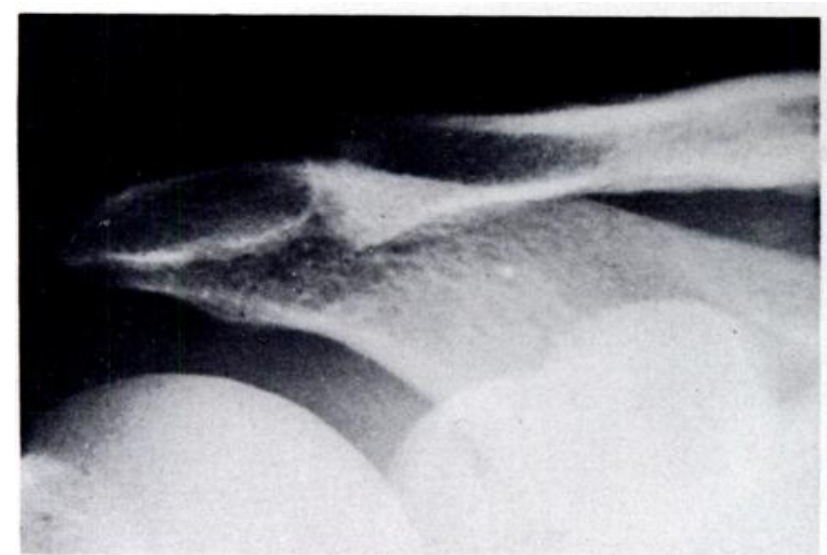

Fig. 6

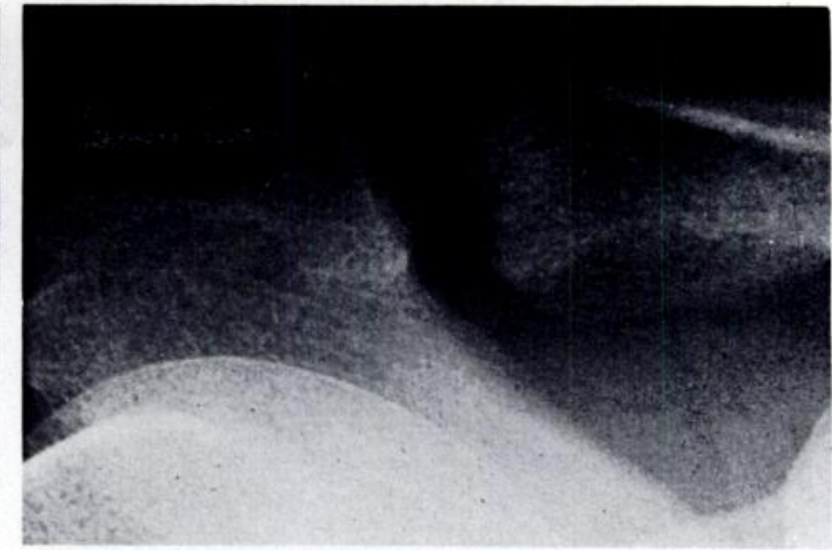

Fig. 7

Standard antero-posterior and 20-degree upward tilt views of the same acromio-clavicular joint (Stripp 1974).

seen. On release of the retractor the fragments fall together and are held with a towel clip for simple interrupted suture of the aponeurotic flaps. Post-operative mobilisation may start on the following day unless a repair of the rotator cuff has been performed.

\section{A CLASSIFICATION OF THE PAINFUL ARC SYNDROME}

All patients had lesions which lay in one of three different parts of the rotator cuff (Table I). The pathology, natural history and prognosis allowed us to classify all patients into one of three distinct types (Table II).

Posterior type-About one-third of all the patients were classified as belonging to the posterior type of painful arc syndrome. All of these responded to treatment by one, two or three injections of local anaesthetic and steroids. Occasionally a second course of treatment was required but none of these progressed to persistent disorder. None required operation.

Anterior type-About one-third of the patients were classified as belonging to the anterior type of painful arc syndrome. Thirty-four out of thirty-six patients in the group recovered after one or two local injections of steroid, with complete resolution. Only two required operation, and in both of these a swollen and inflamed subscapularis tendon seemed to impinge upon the coraco-acromial ligament during abduction and external rotation. Division of the coraco-acromial ligament in these two cases resulted in complete resolution of their symptoms.

Superior type-The remaining third fell into the superior type of our classification. These were the most severe and the most complicated of the cases. Half the patients in this group had the same pathology: degeneration of the acromio-clavicular joint and degeneration of the anterior part of the supraspinatus tendon with usually a small communicating rupture. Ten of the twelve patients were relieved of pain by excision of the outer 1 centimetre of the clavicle, division of the coraco-acromial ligament and simple closure of the underlying defect.
Four of our earliest cases, before we had learnt to distinguish them properly, are particularly instructive: the coraco-acromial ligament was divided in two patients, and they were not relieved; one patient underwent an excision of the outer end of the clavicle which did not relieve his symptoms until it was followed by a second operation to divide the coraco-acromial ligament. In the

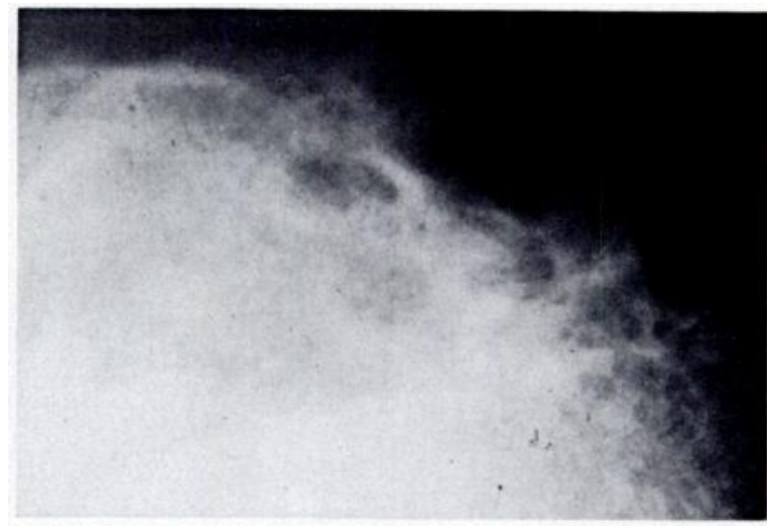

Fig. 8

Tangential view of a biceps sulcus. The marked degenerative changes of osteophytosis, cyst formation and sclerosis can be seen.

fourth case the coraco-acromial ligament only was divided at the first operation and he was not relieved until the outer end of the clavicle had been removed. It became apparent to us that the whole complex of pathological lesions had to be dealt with at the first operation.

A previously unsuspected rupture of the rotator cuff was revealed at operation on five occasions. Since it is our normal practice to carry out arthrography of the shoulder if there is any suspicion of a rotator cuff rupture, it must be said that this group of patients still remains something of a mystery. When a rupture of the rotator cuff was unexpectedly disclosed, we performed a formal repair with a similar result in each case: the preoperative pain for which the patient had sought advice was relieved, but the quality and excursion of movement 
was not improved. This group of patients were more pleased with the operation than we were.

In two patients a small "tumour" was discovered as the cause of the painful arc syndrome. In one of these it had been suspected by the pre-operative study, including suspicious radiographs. Histologically both tumours had features of chronic inflammation of a synovial cyst.

\section{Rupture of the long head of the biceps}

We have made no attempt here to analyse all those cases of rupture of the tendon of the long head of the biceps seen in the shoulder clinic. However, amongst those with a painful arc syndrome we found four patients with rupture of this tendon. In three of the patients this added pathology did not seem to be particularly important,

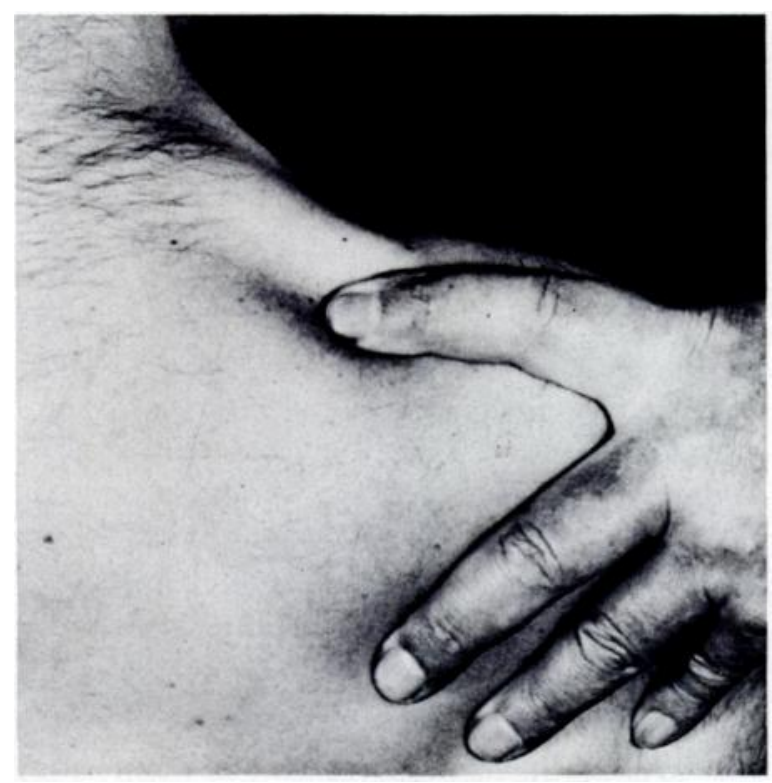

Fio. 9

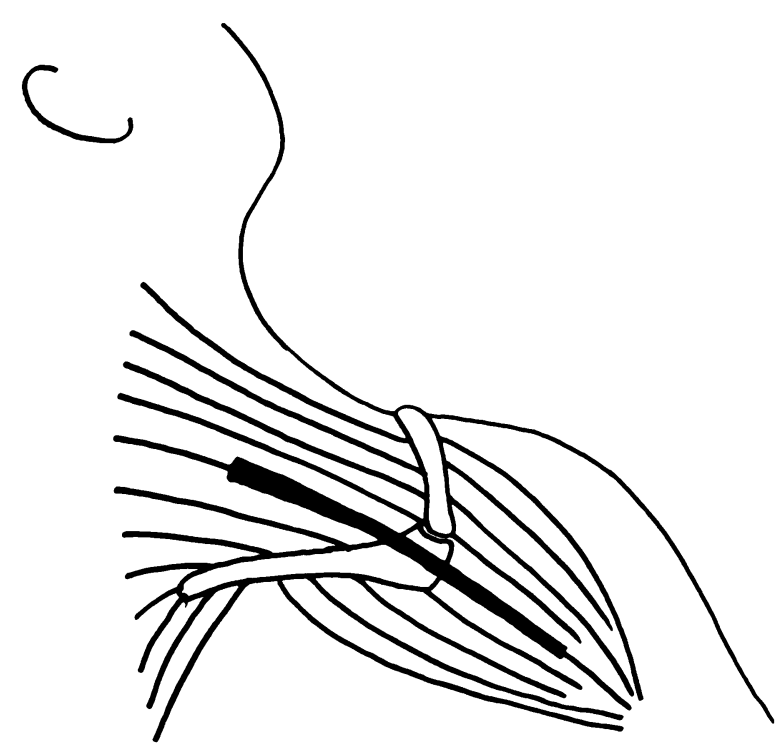

Fig. 10

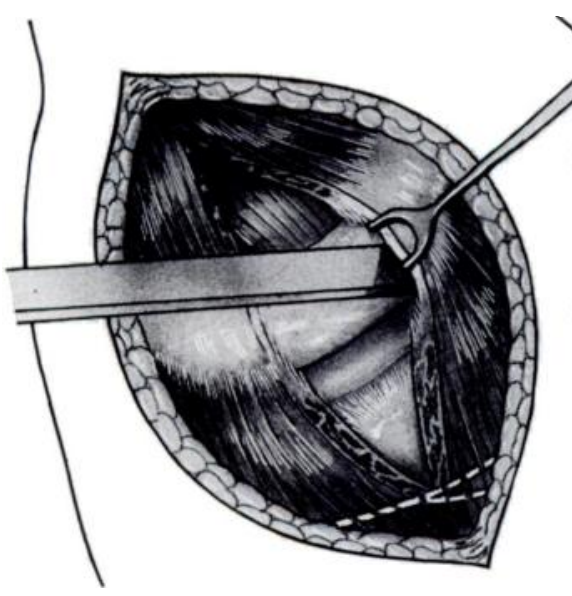

Fig. 11

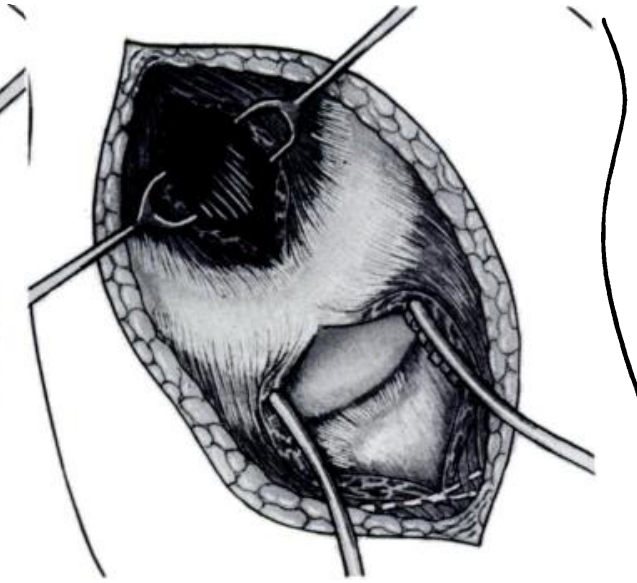

FIG. 12

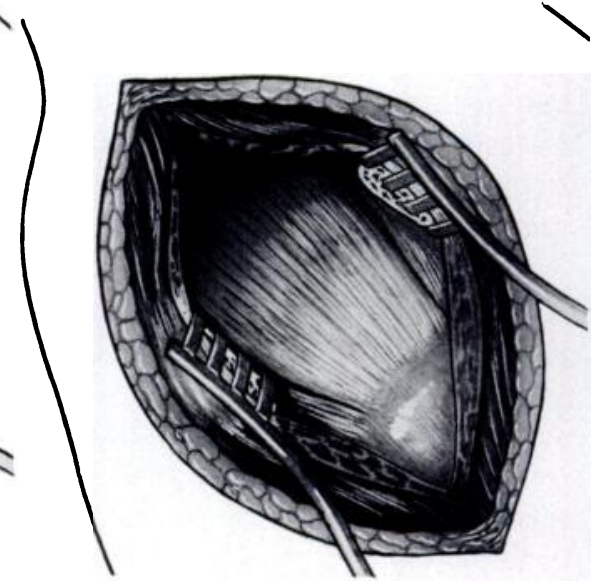

Fig. 13

The transacromial approach. Figure 9-Surface anatomy for the incision: the index finger delineates the fossa between clavicle and spine of the scapula. Figure 10-The line of the incision. Figure 11-Raising the aponeurotic flaps from the acromion. The course of the circumflex nerve is shown. Figure 12-The deltoid has been split and held apart with a self-retaining retractor. The trapezius has also been split and its fibres held apart with two small retractors. The acromion between them is still intact. Figure 13-The acromion has been divided and spread as widely as possible. The pale, intact rotator cuff can be seen.

Nine patients did not, for one reason or another, come to operation and we cannot, therefore, be certain of the underlying pathology. It is interesting to note that in six of these patients who showed radiographic evidence of degeneration of the acromio-clavicular joint, four were not improved by local steroid injections, whereas three patients with normal acromio-clavicular joints were so relieved. apart from indicating general degenerative change. In one patient the proximal part of the tendon was lying free in the joint and was responsible for intermittent pain and instability. Apart from such a rare event we attach no clinical significance (as a guide to management) to the presence or absence of a rupture of the long head of biceps. 
The significance of calcific deposits

We have not here analysed all those patients seen in the shoulder clinic with calcific deposition in one or other painful arc syndrome, calcification in the rotator cuff was present in twelve. A reference to Table II shows that patients with radiographic calcification are scattered,

TABLE I

Differentiation of the Three Common Types of the Painful Arc Syndrome

\begin{tabular}{|c|c|c|c|}
\hline & Posterior & Anterior & Superior \\
\hline Night pain & ++ & ++ & $0+$ \\
\hline Age . . . & $50 ` s$ & 50 's & 40 's \\
\hline Sex ratio & $\mathrm{F}>\mathrm{M}$ & $\mathrm{F}>\mathrm{M}$ & $\mathbf{M}>\mathbf{F}$ \\
\hline Painful arc . . & $60-120$ degrees & $60-120$ degrees & $60-180$ degrees \\
\hline Aggravated by . & Internal rotation & External rotation & Neither \\
\hline Tenderness greatest. & Posterior greater tuberosity & Lesser tuberosity & Greater tuberosity \\
\hline Ruptured LHB & $\mathbf{0}$ & 0 & Occasional \\
\hline A-C joint degenerate & $\mathbf{0}$ & $\mathbf{0}$ & Frequent \\
\hline Prognosis & Very good & Good & Poor without operation \\
\hline
\end{tabular}

TABLE II

Analysis of All Cases

\begin{tabular}{|c|c|c|c|c|c|c|}
\hline Type & $\begin{array}{l}\text { Number } \\
\text { of cases }\end{array}$ & Calcification & A-C Joint & Pathology & Treatment & Result \\
\hline Posterior & 32 & $\begin{array}{l}\text { Supraspinatus } 1 \\
\text { Infraspinatus } 1\end{array}$ & Normal & Not seen & Local steroids & Resolution \\
\hline \multirow[t]{2}{*}{ Anterior } & 34 & $\begin{array}{l}\text { Supraspinatus } 3 \\
\text { Infraspinatus } 1 \\
\text { Subscapularis } 1\end{array}$ & Normal & Not seen & Local steroids & Resolution \\
\hline & 2 & 0 & Normal & $\begin{array}{l}\text { Swollen, inflamed } \\
\text { subscapularis tendon }\end{array}$ & $\begin{array}{l}\text { Division of coraco- } \\
\text { acromial ligament }\end{array}$ & Resolution \\
\hline \multirow[t]{7}{*}{ Superior } & 10 & $\begin{array}{l}\text { Supraspinatus } 3 \\
\text { Subscapularis } 1\end{array}$ & Degenerate & $\begin{array}{l}\text { Frayed anterior part of } \\
\text { supraspinatus; small } \\
\text { rupture; in two cases } \\
\text { ruptured long head of } \\
\text { biceps* }\end{array}$ & $\begin{array}{l}\text { Excision outer } 1 \mathrm{~cm} \text {. } \\
\text { clavicle; division of } \\
\text { coraco-acromial } \\
\text { ligament; simple } \\
\text { closure of tear }\end{array}$ & Painful arc relieved \\
\hline & 2 & 0 & Degenerate & $\begin{array}{l}\text { Frayed anterior part of } \\
\text { supraspinatus; small } \\
\text { rupture }\end{array}$ & $\begin{array}{l}\text { Division of coraco- } \\
\text { acromial ligament } \\
\text { only }\end{array}$ & No improvement \\
\hline & 1 & 0 & Normal & $\begin{array}{l}\text { Frayed anterior part of } \\
\text { supraspinatus; small } \\
\text { rupture + rupture LHB }\end{array}$ & $\begin{array}{l}\text { Division of coraco- } \\
\text { acromial ligament } \\
\text { only }\end{array}$ & Painful arc relieved \\
\hline & 5 & Supraspinatus 1 & Degenerate & $\begin{array}{l}\text { Massive rupture of } \\
\text { rotator cuff }\end{array}$ & Reconstruction/repair & $\begin{array}{l}\text { Pain relieved, } \\
\text { movement not } \\
\text { improved }\end{array}$ \\
\hline & 2 & 0 & Normal & Synovial cyst & Excision & Painful arc relieved \\
\hline & 6 & 0 & Degenerate & $\begin{array}{l}\text { Not seen } \\
\text { (one ruptured LHB) }\end{array}$ & Local steroids & $\begin{array}{l}4 \text { not improved. } \\
2 \text { painful arcs } \\
\text { relieved }\end{array}$ \\
\hline & 3 & 0 & Normal & Not seen & Local steroids & Painful arc relieved \\
\hline
\end{tabular}

* In one of the cases of ruptured long head of biceps the proximal stump behaved like a loose body in the shoulder.

part of the rotator cuff. The clinical picture of acute calcific deposit in the shoulder is quite different to that of the painful arc syndrome with which it should not be confused. In our series of ninety-seven patients with a apparently at random, throughout the three types. In only six cases did the area of calcification correspond in site to the source of the patient's complaint at the time of consultation. Whereas the presence of calcification on 
the radiograph in a patient with a painful arc syndrome must of course be carefully taken into account, it should not be assumed that the calcified area is necessarily the source of symptoms. The tendency of the supraspinatus tendon to harbour most calcific deposits (De Palma and Kruper 1961) around the shoulder may be related to its relatively poor blood supply when compared with the other structures surrounding the cuff. In this study the demonstration of the source of the patients' symptoms indicated that the calcific deposits seen in the radiographs were usually not the source of the painful arc, but that this arose from a non-calcified lesion nearby.

\section{DISCUSSION}

We are not aware of any previous work which has attempted to classify patients with a painful arc syndrome and comparison of our results with those recorded in other papers is therefore invalid. It is however necessary to advance some hypothesis to explain the different behaviour of the three types. Posterior lesions, the result of either trauma or degeneration or a combination of both, lie in well-vascularised muscle. Impingement against the acromion during abduction varies with rotation of the humerus and the lesion may be cleared by outward rotation of the humerus during abduction. Conditions for healing are good, and the repair of the lesion is unhindered.

The anterior lesions of the subscapularis tendon lie also in well-vascularised muscle. However, in abduction and flexion the tendon impinges beneath the coracoacromial ligament. Healing of these lesions is hindered by repeated impingement and the prognosis is corres- pondingly somewhat poorer than for posterior cuff lesions.

In the superior type of lesion the supraspinatus tendon and the nearby long head of biceps tendon are poorly vascularised (Macnab 1973) and are confined beneath the coraco-acromial arch. In both abduction and flexion the lesion lies directly beneath the acromioclavicular joint (Neer 1972). In cases of degeneration of this joint there is encroachment on the space available beneath the arch due to periarticular swelling. This must considerably reduce the chance of spontaneous healing and probably explains the poor prognosis in this group.

\section{CONCLUSIONS}

It appears that a firm prognosis and a guide to treatment in cases of the painful arc syndrome may be obtained after examination at the patient's first attendance: 1) Those with lesions of the posterior part of the rotator cuff will respond to non-operative treatment. 2) Of those with anterior lesions and no radiological abnormality of the acromio-clavicular joint the majority will respond to non-operative treatment, but a few may require division of the coraco-acromial ligament. 3) Those who suffer from the superior type of painful arc syndrome, usually with degeneration of the acromio-clavicular joint, will probably require operation to excise the outer 1 centimetre of the clavicle, divide the coraco-acromial ligament, and repair any underlying defect of the rotator cuff.

There remain but a few causes of the painful arc syndrome which require careful analysis and treatment on their individual merit.

\section{REFERENCES}

Adams, J. C. (1955) Outline of Orthopaedics. First Edition, p. 217. London and Edinburgh: E. and S. Livingstone.

Armstrong, J. R. (1949) Excision of the acromion in treatment of the supraspinatus syndrome. Journal of Bone and Joint Surgery, 31-B, 436-442.

Bosworth, D. M. (1940) An analysis of twenty-eight consecutive cases of incapacitating shoulder lesions, radically explored and repaired. Journal of Bone and Joint Surgery, 22, 369-392.

Burns, B. H., and Ellis, V. H. (1937) Recent Advances in Orthopaedic Surgery, p. 151. London: J. and A. Churchill Ltd.

Codman, E. A. (1934) The Shoulder. Boston: Privately printed.

Debeyre, J., Patte, D., and Elmelik, E. (1965) Repair of ruptures of the rotator cuff of the shoulder. Journal of Bone and Joint Surgery, 47-B, 36-42.

DePalma, A. F., and Kruper, J. S. (1961) Long-term study of shoulder joints afflicted with and treated for calcific tendinitis. Clincial Orthopaedics, 20, 61-72.

Diamond, B. (1964) The Obstructing Acromion. Springfield, Illinois: Charles C. Thomas.

Fowler, E. B. (1932) Rupture of spinati tendons and capsule, repaired by a new operation. Illinois Medical Journal, 61 332-334.

Kessel, L. (1967) Orthopaedics. In Clinical Surgery. Number 13, p. 403. Edited by G. C. Lloyd-Roberts. London: Butterworths.

McLaughlin, H. L. (1945) Lesions of the musculotendinous cuff of the shoulder. 2. Differential diagnosis of rupture. Journal of the American Medical Association, 128, 563-568.

McFarland, B., and Osborne, G. (1954) Approach to the hip. Journal of Bone and Joint Surgery, 36-B, 364-367.

Macnab, I. (1973) Rotator cuff tendinitis. Annals of the Royal College of Surgeons of England, 53, 271-287. Neer, C. S. II. (1972) Anterior acromioplasty for the chronic impingement syndrome in the shoulder. Journal of Bone and Joint Surgery,
54-A, 41-50.

Stripp, W. J. (1974) Personal communication.

Trickey, E. L. (1974) Personal communication. 